\title{
High-yield fabrication of perpendicularly magnetised synthetic antiferromagnetic nanodiscs
}

\author{
Emma N. Welbourne $(\bowtie)$, Tarun Vemulkar, and Russell P. Cowburn \\ Department of Physics, Cavendish Laboratory, University of Cambridge, J. J. Thomson Avenue, Cambridge, CB3 OHE, UK
}

(C) The Author(s) 2021

Received: 1 October 2020 / Revised: 8 December 2020 / Accepted: 25 December 2020

\begin{abstract}
Synthetic antiferromagnetic (SAF) particles with perpendicular anisotropy display a number of desirable characteristics for applications in biological and other fluid environments. We present an efficient and effective method for the patterning of ultrathin Ruderman-Kittel-Kasuya-Yoshida coupled, perpendicularly magnetised SAFs using a combination of nanosphere lithography and ion milling. A Ge sacrificial layer is utilised, which provides a clean and simple lift-off process, as well as maintaining the key magnetic properties that are beneficial to target applications. We demonstrate that the method is capable of producing a particularly high yield of well-defined, thin film based nanoparticles.
\end{abstract}

\section{KEYWORDS}

nanodiscs, synthetic antiferromagnets, perpendicular anisotropy, sacrificial layers, nanofabrication

\section{Introduction}

Magnetic particles are widely used in a variety of biomedical applications, including cancer therapy, drug delivery and as contrast agents $[1,2]$. Characteristics that are highly beneficialand as such formulate the "ideal particle" for many biological or liquid applications - consist of a variable and high saturation magnetisation, sharp and tunable switching for efficient access to a desired saturated state, an easy axis of magnetisation with a high anisotropy to allow the efficient transduction of torque under applied field, and a zero remanence state with a low susceptibility around zero field preventing agglomeration [3]. Superparamagnetic nanoparticles (SPNPs) have so far been the major candidates in biological applications, particularly in hyperthermia for cancer therapy, however their simple magnetic properties come with limitations. Despite their zero remanence state, they exhibit considerable coercivity at higher field sweep rates. They are also prone to agglomeration via surface interactions, due to their colloidal state, without significant engineering [4-7]. Furthermore, their magnetic properties do not allow any tunability in switching field and an increase in moment can lead to a change of magnetic state, leading to ferromagnetic particles. Perpendicularly magnetised (PM) synthetic antiferromagnets (SAFs) have been shown to overcome the limitations of SPNPs and have all of the desirable properties described for an ideal particle in biological applications, offering much finer control over activation and assembly [3]. They have been demonstrated as strong candidates for the magnetomechanical destruction of cancer cells [8] and their self-assembly behaviour in liquid could be interesting in areas such as microfluidics, biosensing or soft robotics [9]. Additionally, highaspect ratio particles, such as discs, have been shown to promote cellular endocytosis $[10,11]$, which is highly advantageous for in vitro applications.
So far, it has been challenging to produce a high-yield fabrication process for thin film based particles, such as SAFs, with sub-micron dimensions. Optical lithography, though a powerful and efficient method for patterning, is diffraction limited in the sub-micron regime. Furthermore, the previously used method of growing thin film stacks on top of photoresist pillars created magnetic microdiscs with significant shape defects that severely limited their aspect ratio [3]. The prevailing method, which made the production of film based nanoparticles possible, is hole-mask colloidal lithography (HMCL) [12, 13]. This method includes a multi-stage deposition and etching process, and does not lead to the production of a competitive particle yield. An alternative method for the production of such particles is nanoimprint lithography $[14,15]$, which has previously been used to produce in-plane SAF nanodiscs $[16,17]$. However, this method suffers from similar constraints as HMCL, including the fact that deposition of a PM thin film into a nanoscale hole may have significantly negative effects on both magnetic properties and particle shape, which is due to shadowing effects during physical vapour deposition.

Here we detail an updated version of the cost and timeeffective fabrication method of nanosphere lithography with ion milling [18], and make use of a Ge sacrificial layer [13], to demonstrate more optimal patterning and production of SAF nanodiscs. The method uses a streamlined protocol, with the use of polystyrene (PS) beads as a direct lithography mask, allowing access to the nanoscale. Particle yield is increased by spin coating the PS beads [19], rather than the dropcast application used in the HMCL method [13]. Additionally, the spin coating method is commercially viable, easily scalable and simple to tune for particle density. By starting with a continuous thin film and using ion milling to form the discs, we achieve excellent pattern transfer of the two-dimensional (2D) projection of the PS beads' spherical shape onto the film. 
Finally, the choice of $\mathrm{Ge}$ as a sacrificial layer ensures we conserve our carefully engineered magnetic properties, while facilitating ease of lift-off. These well-defined, magnetic nanodiscs are better optimised for biological applications, particularly in vitro therapies, such as the magneto-mechanical destruction of cancer cells.

\section{Results and discussion}

\subsection{Thin films and sacrificial layers}

The typical motif of a PM SAF multilayer thin film is $\mathrm{Ta} / \mathrm{Pt} / \mathrm{CoFeB} / \mathrm{Pt} / \mathrm{Ru} / \mathrm{Pt} / \mathrm{CoFeB} / \mathrm{Pt}$, as shown in Fig. $1(\mathrm{a})$. The easy axis response of such a film is shown in the magnetooptical Kerr effect (MOKE) loop in Fig. 1(b), with the arrows representing the magnetisation direction of the $\mathrm{CoFeB}$ layers. The PM state of the system is created by spin-orbit interactions between the $\mathrm{Pt}$ and $\mathrm{CoFeB}$ [20]. This state has been demonstrated as key to effective transduction of torque [8], which is highly beneficial in applications that involve mechanical actuation. The antiparallel state at remanence and low magnetic fields is induced by Ruderman-Kittel-Kasuya-Yoshida (RKKY) coupling [22-24], provided by a Ru interlayer with a thickness that lies at an antiferromagnetic coupling peak [25]. This produces a zero remanence state and low susceptibility, which prevents particle agglomeration [3, 26, 27]. The hysteresis loop in Fig. 1(b) has an apparent non-zero magnetisation due to the depth dependence of the MOKE signal. The Pt interlayers either side of the Ru not only stabilise the PM state [21], but also yield a means of tuning the sharp switch to the saturated, parallel state [28]. This allows a film to be tailored to a specific application [3].

As the magnetic state of our PM SAF films is governed by interfacial interactions, the RKKY coupling and spin-orbit coupling, it is very sensitive to changes in growth conditions. Hence, the choice of sacrificial layer in a thin film based particle fabrication method must be made based on two key criteria: ease of removal, and therefore the ability to release particles into solution, and the effect of the underlayer on the magnetic properties of a thin film grown on top. Two key features to conserve, which we noted as desirable for magnetic particles to be used in biological applications, are the sharp magnetic transitions and zero remanence state.

Conventionally, photoresist is implemented as a sacrificial layer as it provides simplicity in both application and removal. However, exposing photoresist to ion milling commonly causes it to crosslink, making it significantly challenging to dissolve. Thus, $\mathrm{Ge}$ and $\mathrm{Al}$ were chosen as suitable candidates: They are amenable to the same deposition methods as the thin film, therefore simplifying the process requirements. Additionally, ease of lift-off is not compromised, as they readily dissolve in
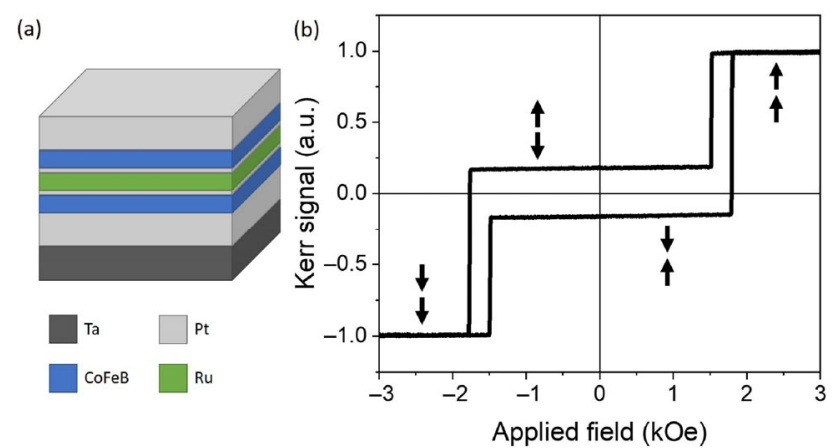

Figure 1 (a) A schematic of the typical PM SAF thin film multilayer stack. (b) An easy axis MOKE hysteresis loop of a PM SAF thin film. hydrogen peroxide (Ge) and basic solvents ( $\mathrm{Al})$, thus eliminating the use of the potentially toxic, organic solvents that are required for crosslinked photoresist. Here, Ge has an advantage over $\mathrm{Al}$ as a sacrificial layer, as hydrogen peroxide naturally degrades to water: This eliminates extra washing steps of lifted off particles, limiting the loss of yield.

To investigate the effect that each underlayer has on the hysteresis of PM CoFeB, sputtered magnetic stacks were grown on evaporated $\mathrm{Ge}$, sputtered $\mathrm{Al}$, and on $\mathrm{Si}$ to compare. PM SAF stacks were grown in the form $\mathrm{Ta}(2) / \mathrm{Pt}(4) / \mathrm{CoFeB}(0.9) /$ $\mathrm{Pt}\left(t_{\mathrm{Pt}}\right) / \mathrm{Ru}(0.9) / \mathrm{Pt}\left(t_{\mathrm{Pt}}\right) / \mathrm{CoFeB}(0.9) / \mathrm{Pt}(4) / \mathrm{Ta}(2)$ (thicknesses in $\left.\mathrm{nm}\right)$. Pt thickness, $t_{\mathrm{Pt}}$, was varied from $0.41-0.69 \mathrm{~nm}$ to create a series of SAFs with a range of coupling strengths [21, 28]. Single layers of $0.9 \mathrm{~nm} \mathrm{PM} \mathrm{CoFeB}$ were also grown on each substrate to further analyse the effect of the underlayers on the magnetic transitions, the data for these samples is presented in Fig. S2 in the Electronic Supplementary Material (ESM).

\subsection{Effect of underlayer on SAF hysteresis}

Figures 2(a)-2(c) show the easy axis MOKE loops of the PM SAF series. We see a clear, drastic effect on the behaviour of the films grown on Al. The sharpness of the transitions is completely lost and the coercivity has increased. This leads to a loss of the zero remanence state in the more weakly coupled films. In comparison, the Ge underlayer has a much smaller effect on the hysteresis loops. We observe a small decrease in saturation field across the series, however, there is a relatively small change in coercivity and the sharpness of the transitions is conserved. The MOKE data indicates that $\mathrm{Ge}$ is a good option for a sacrificial layer, as it appears to ensure the robustness of magnetic properties and also maintains the access to a large range of coupled films with zero remanence and different switching fields.

Domain imaging of the thin films, using polar Kerr microscopy, reveals the nucleation and propagation dynamics that drive the MOKE loop transitions of the PM SAFs. The significantly sloped transitions seen in the loops of the samples grown on $\mathrm{Al}$ underlayers are the result of nucleation dominant switching, where the domain formations are too small to observe with the polar MOKE imaging. Conversely, the sharp transitions seen for the films grown on $\mathrm{Si}$ and $\mathrm{Ge}$ translate into clear domain images with fast moving domain walls. Figures $2(\mathrm{~d})$ and $2(\mathrm{e})$ show domain images of the $t_{\mathrm{Pt}}=$ $0.5 \mathrm{~nm} \mathrm{SAF}$, on both $\mathrm{Si}$ and Ge, during the antiparallel to parallel (AP-P) transition. These images show a number of nucleation sites, creating small domains that merge together. This is consistent with previous findings on the domain dynamics of coupled CoFeB: The AP-P transition involves a higher density of nucleation sites for more highly coupled samples [29]. We observe rapid domain wall propagation, which results in the sharp transitions in the corresponding MOKE loops.

Atomic force microscopy (AFM) measurements offer further explanation of the effects that the sacrificial layers have on the magnetic stacks. Figure 3(a) shows that the roughness of $\mathrm{Al}$ is considerably higher than that of Si or Ge. Additionally, when we look at the radial point spectral density function (PSDF) profiles in Fig. 3(b), there is a significant magnitude of roughness in the $\mathrm{Al}$ sample in the $10-20 \mathrm{~nm}$ range, which is the lengthscale associated with domain wall dynamics in PM thin films [30]. This leads to a significant effect on the nucleation and pinning of domain walls during magnetic reversal, and hence more sloped transitions in the hysteresis loops. Ge does not exhibit significant roughness on the same length-scale, with values comparable to that of $\mathrm{Si}$, therefore it has a much smaller effect on the hysteresis loops of these thin films. These findings 

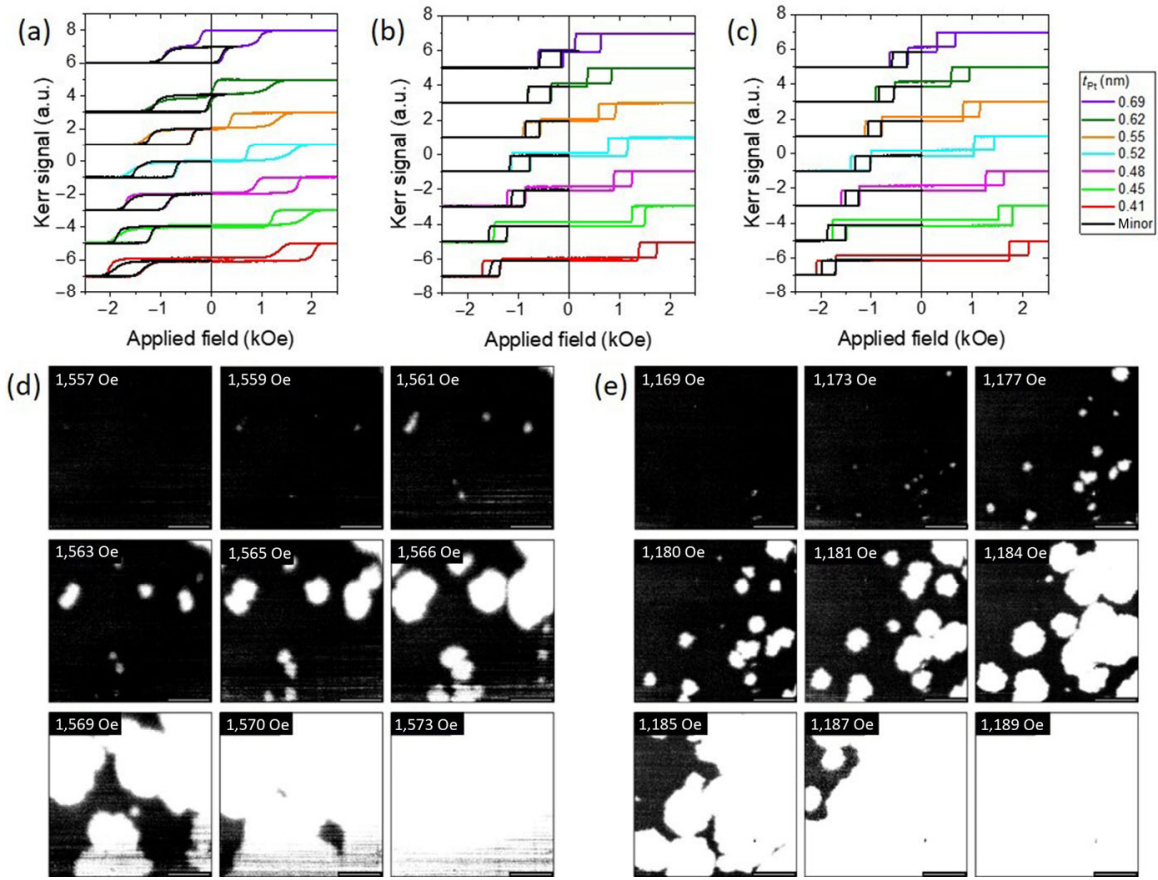

Figure 2 MOKE loops of SAF thin films grown on (a) Al, (b) Ge and (c) Si. (d) Kerr microscopy domain images of SAFs switching AP-P for bilayers grown on (a) Si and (b) Ge. The Pt interlayer thickness is $0.5 \mathrm{~nm}$. Scale bar is $100 \mu \mathrm{m}$.

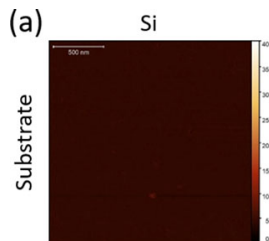

(a)

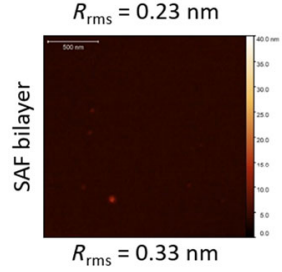

(b)

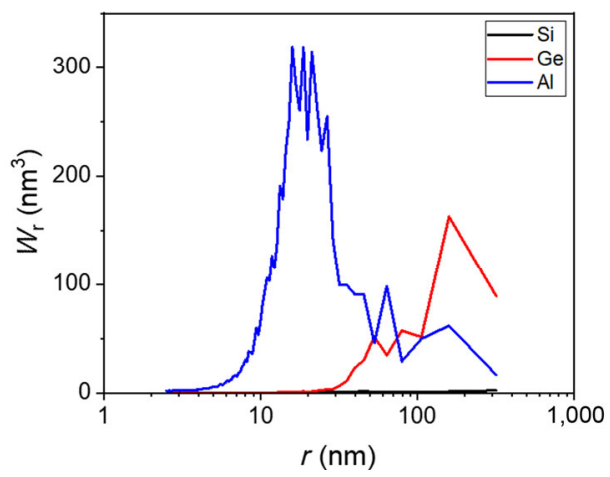

Figure 3 (a) AFM topography images of the substrates, $\mathrm{Si} / \mathrm{Ge} / \mathrm{Al}$, and of the top Ta layer of a SAF bilayer $\left(t_{\mathrm{pt}}=0.69 \mathrm{~nm}\right)$ grown on top of each substrate. The root mean squared roughness $\left(R_{\mathrm{rms}}\right)$ is noted below each image. (b) The radial PSDF profiles taken from each of the substrate scans in (a), where $r$ is radius and $W_{\mathrm{r}}$ is the radial PSDF, representing roughness at different length-scales.

support Ge as a suitable material for the sacrificial layer in the production of thin film based nanoparticles.

\subsection{Fabrication of nanodiscs}

A schematic of the nanodisc fabrication process is displayed in Fig. 4. Corresponding scanning electronic microscopy (SEM) images of three of the key stages are included in Fig. S3 in the ESM. The process consists of four main stages: material deposition of the sacrificial layer, SAF stack and a capping layer; nanosphere lithography [19] of PS beads to form a mask; ion milling to pattern the thin film into discs; and finally, liquid etches to lift the magnetic nanodiscs into solution. First, (1) Ge $(\sim 50 \mathrm{~nm})$ is evaporated onto a Si substrate, forming a sacrificial layer that will later allow the nanodiscs to be lifted into solution. (2) The PM SAF stack is sputtered on top, in the form $\mathrm{Ta} / \mathrm{Pt} / \mathrm{CoFeB} / \mathrm{Pt} / \mathrm{Ru} / \mathrm{Pt} / \mathrm{CoFeB} / \mathrm{Pt} / \mathrm{Ta}$. (3) A capping layer of $\mathrm{Al}$, deposited by either sputtering or evaporation, is added. This promotes the adhesion of PS beads and also provides a secondary sacrificial layer, which aids the removal of material that is redeposited during ion milling. (4) PS beads (Polybead ${ }^{\mathbb{B}}$ microspheres, diameter of $100 \mathrm{~nm}-1 \mu \mathrm{m}$ ), which have been diluted in deionised water as necessary (see Table 1), are spin coated across the Al surface to create a dense monolayer. (5) Oxygen plasma ashing is used to partially shrink the PS

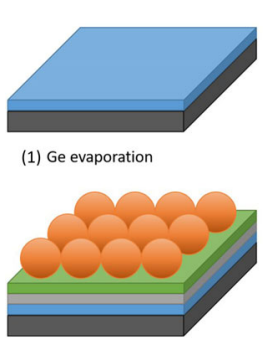

(4) PS bead spin coating

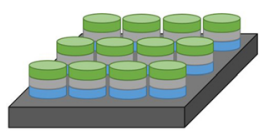

(7) Sonication

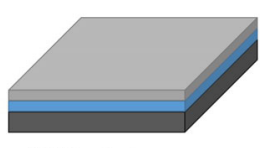

(2) SAF sputtering

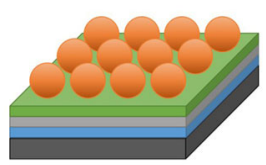

(5) $\mathrm{O}_{2}$ plasma ash

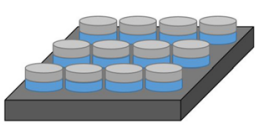

(8) Al etch

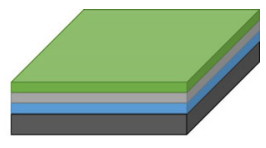

(3) Al deposition

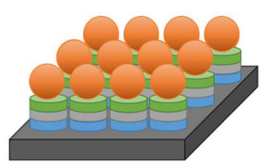

(G) Ion milling

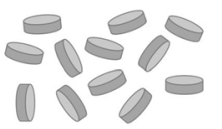

(9) Ge etch
Figure 4 (1) Ge is evaporated onto Si. (2) The SAF stack is sputtered on top of the Ge. (3) An Al layer is deposited on top of the SAF. (4) PS beads are spin coated across the surface. (5) Plasma ashing creates gaps between PS beads. (6) The PS beads are used as an ion milling mask and discs of the Ge-SAF-Al stack are made. (7) Sonication in water removes the PS beads. (8) An Al etch removes the cap. (9) A Ge etch lifts off the nanodiscs into solution. 
Table 1 Dilution (beads:water) and plasma ashing time criteria for the PS beads of different diameters

\begin{tabular}{ccccc}
\hline Diameter $(\mathrm{nm})$ & 100 & 200 & 500 & 1,000 \\
Dilution & $2: 1$ & $1: 1$ & $1: 0$ & $1: 0$ \\
Ash time (s) & 30 & 60 & 240 & 300 \\
\hline
\end{tabular}

beads. The timing of the ashing is carefully tuned to minimise the size reduction and prevent bead deformation, while creating sufficient spacing between beads for subsequent film patterning into individual discs (see Table 1). (6) The PS beads are used as a lithography mask during the Ar ion milling of the full thin film stack, transferring the $2 \mathrm{D}$ projection of the beads onto the film beneath. (7) The PS beads are removed by sonication in water. (8) The sample is briefly soaked $(\sim 30 \mathrm{~s})$ in photoresist developer (a tetramethylammonium hydroxide based solvent), which etches away the $\mathrm{Al}$ cap and leaves behind just the SAF discs on the substrate. Finally, (9) the sample is immersed in a $35 \% \mathrm{H}_{2} \mathrm{O}_{2}$ solution for at least $10 \mathrm{~min}$, which etches away the Ge sacrificial layer. It is then submerged in water, diluting away the $\mathrm{H}_{2} \mathrm{O}_{2}$, and sonicated to lift-off the discs into solution. This process leaves behind a clean, reusable Si wafer, which is highly beneficial from the perspectives of reducing production costs or limiting consumption of resources.

\subsection{Characterisation of nanodiscs}

This fabrication technique has so far been demonstrated using PS beads of diameters between $100 \mathrm{~nm}$ and $1 \mu \mathrm{m}$, as shown in Fig. 5. In these images we see that the use of plasma ashing, to
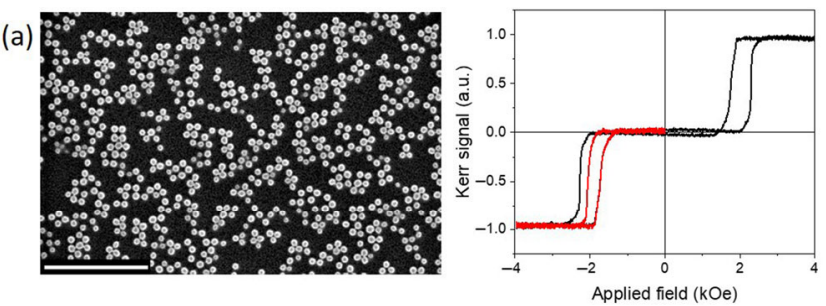

(b)
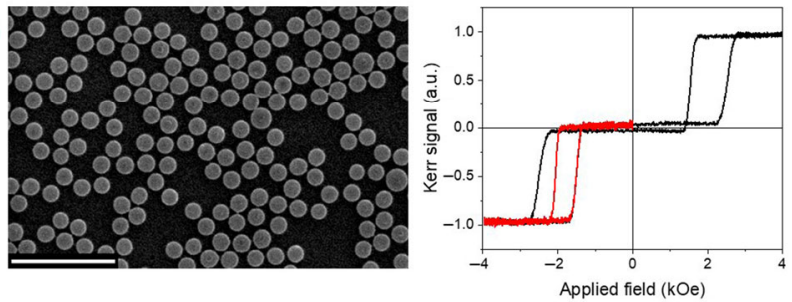

(c)
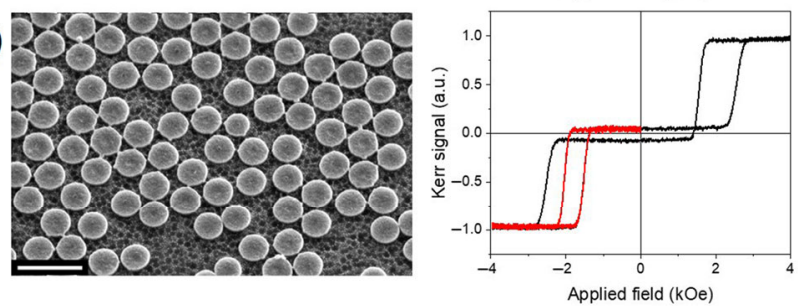

(d)
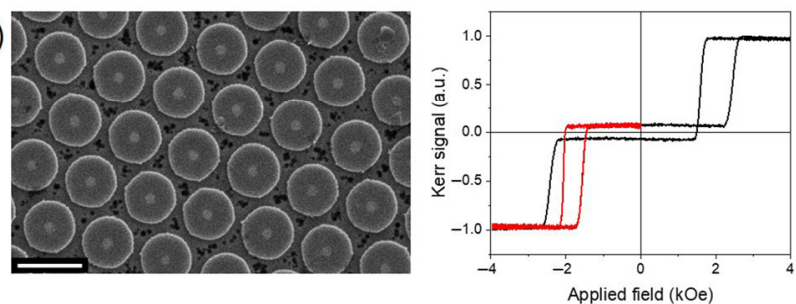

Figure 5 SEM images of SAF discs made with PS beads of diameters (a) $100 \mathrm{~nm}$, (b) $200 \mathrm{~nm}$, (c) $500 \mathrm{~nm}$ and (d) $1 \mu \mathrm{m}$. All scale bars are $1 \mu \mathrm{m}$. Alongside are major (black) and minor (red) MOKE loops taken of discs of each size. separate out the PS beads prior to milling, produces individual discs with diameters slightly under that of the original beads. It is noted that a small point is observed at the centre of some of the nanodiscs. This small feature originates from the fabrication process, as detailed in Fig. S4 in the ESM.

Yield calculations show percentages of coverage by area that are significantly higher than those previously reported, for all disc sizes: with the $100 \mathrm{~nm}$ beads we achieved $31 \%$ coverage $\left(\sim 80 \times 10^{6}\right.$ discs $\left./ \mathrm{mm}^{2}\right)$, with $200 \mathrm{~nm}$ beads $41 \%$ coverage $\left(\sim 18 \times 10^{6} \mathrm{discs} / \mathrm{mm}^{2}\right)$, with $500 \mathrm{~nm}$ beads $55 \%$ coverage $\left(\sim 4 \times 10^{6}\right.$ discs $\left./ \mathrm{mm}^{2}\right)$ and with $1 \mu \mathrm{m}$ beads $50 \%$ coverage $\left(\sim 8 \times 10^{5} \mathrm{discs} / \mathrm{mm}^{2}\right)$. For comparison, previous HMCL of $\sim 100 \mathrm{~nm}$ discs achieved around $10 \%$ coverage, yielding just a third of the number produced with this new method [13]. Furthermore, the optical lithography based, photoresist pillar method for making $2 \mu \mathrm{m}$ discs gives $~ 30 \%$ coverage. Therefore, our nanosphere lithography is highly competitive with the traditional optical lithography technique in coverage. As our sacrificial layer is amenable to the same deposition methods as our magnetic stack, there is additional scope for a three-dimensional (3D) increase in particle density through the insertion of Ge buffers between multiple SAF layers.

$500 \mathrm{~nm}$ discs fabricated on $\mathrm{Si}$ and Ge were compared. The thin film stack grown for patterning is described by: $\mathrm{Ta}(2) /$ $\mathrm{Pt}(2) / \mathrm{CoFeB}(0.9) / \mathrm{Pt}(0.3) / \mathrm{Ru}(0.9) / \mathrm{Pt}(0.3) / \mathrm{CoFeB}(0.9) / \mathrm{Pt}(2) / \mathrm{Ta}(2)$ (thicknesses in $\mathrm{nm}$ ). MOKE loops of each size of particle, taken before lift-off, are displayed alongside each of the SEM images in Fig. 5. There is a decrease in coupling and an increase in coercivity for all of the disc samples compared with an equivalent thin film, however, there is no significant difference in these parameters between the different disc sizes.

To test the robustness of the new fabrication method, a set of $1 \mu \mathrm{m}$ discs (see Fig. 6(a)) were created from a more complex thin film of the form: $\mathrm{Ta}(2)[/ \mathrm{Pt}(2) / \mathrm{CoFeB}(0.9) /$ $\left.\mathrm{Pt}\left(t_{\mathrm{Pt}_{\mathrm{t}}}\right) / \mathrm{Ru}(0.9) / \mathrm{Pt}\left(t_{\mathrm{Pt}}\right) / \mathrm{CoFeB}(0.9) / \mathrm{Pt}(2) / \mathrm{Ta}(2)\right]_{2}$ (thicknesses in $\mathrm{nm})$ where $t_{\mathrm{Pt}}=0.45$ and $0.3 \mathrm{~nm}$. Figures $6(\mathrm{~b})-6(\mathrm{~d})$ present vibrating sample magnetometry (VSM) measurements of the original film, and the discs before and after lift-off into liquid. The distinct series of magnetic transitions is maintained from the thin film into the discs on chip. Additionally, we can still distinguish between the independent switches in the liquid response. The ability to resolve the separate transitions after both patterning and lift-off strongly supports the use of the (a)
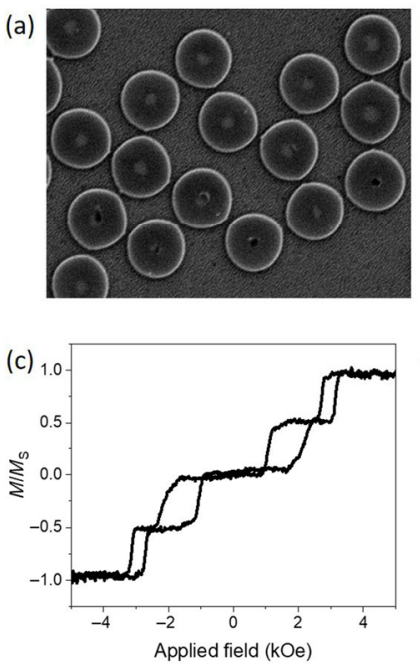

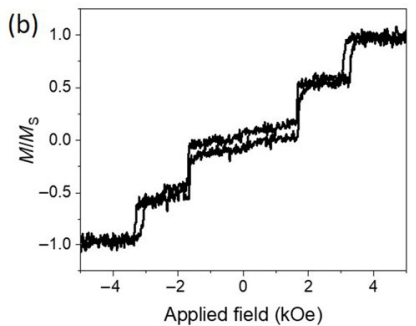

(d) 1.0

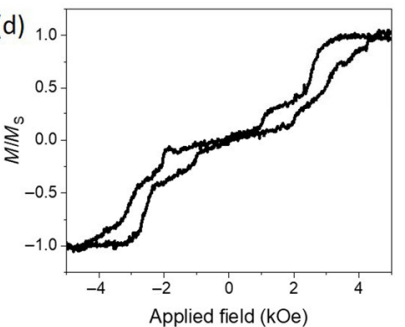

Figure 6 (a) An SEM image of $1 \mu \mathrm{m}$ discs patterned from a double SAF thin film with a $1 \mu \mathrm{m}$ scale bar. VSM measurements of (b) the film, and of the discs (c) before and (d) after lift-off into water. 
new fabrication method for making particles, including for more complex magnetic systems. Furthermore, the particle behaviours here show that we can maintain access to multiple switches in a single sample, and indicate that this would also be possible for a particle mixture, which could be beneficial in a variety of applications such as microfluidic devices and cell sorting.

\section{Conclusions}

We have presented an efficient and effective method for the fabrication of thin film based nanodiscs. A Ge sacrificial layer has been shown to ensure the robustness of a PM SAF film, without compromising ease of lift-off. PS beads have been used in the updated combination of nanosphere lithography with ion milling, which is capable of producing a particularly high yield of high-aspect ratio nanodiscs. This method is widely applicable to the production of nanoparticles from other relevant thin films, such as magnetic vortices or in-plane SAFs. The production of these types of particles is interesting for liquid applications, particularly in biomedical research areas, and provides a nanoscale alternative to the SAF microdiscs that have previously been implemented in magneto-mechanical destruction cancer therapy.

\section{Acknowledgements}

This work was supported by the European Research Council (No. 779822).

Electronic Supplementary Material: Supplementary material (experimental methods for film growth, lithography and sample characterisation; additional MOKE data and domain images; additional schematics and SEM images of lithography process) is available in the online version of this article at https://doi.org/10.1007/s12274-021-3307-1.

Open Access This article is licensed under a Creative Commons Attribution 4.0 International License, which permits use, sharing, adaptation, distribution and reproduction in any medium or format, as long as you give appropriate credit to the original author(s) and the source, provide a link to the Creative Commons licence, and indicate if changes were made.

The images or other third party material in this article are included in the article's Creative Commons licence, unless indicated otherwise in a credit line to the material. If material is not included in the article's Creative Commons licence and your intended use is not permitted by statutory regulation or exceeds the permitted use, you will need to obtain permission directly from the copyright holder.

To view a copy of this licence, visit http://creativecommons.org/licenses/by/4.0/.

\section{References}

[1] Pankhurst, Q. A.; Connolly, J.; Jones, S. K.; Dobson, J. Applications of magnetic nanoparticles in biomedicine. J. Phys. D Appl. Phys. 2003, 36, R167-R181.

[2] Pankhurst, Q. A.; Thanh, N. T. K.; Jones, S. K.; Dobson, J. Progress in applications of magnetic nanoparticles in biomedicine. J. Phys. $D$ Appl. Phys. 2009, 42, 224001.

[3] Vemulkar, T.; Mansell, R.; Petit, D. C. M. C.; Cowburn, R. P.; Lesniak, M. S. Highly tunable perpendicularly magnetized synthetic antiferromagnets for biotechnology applications. Appl. Phys. Lett. 2015, 107, 012403.

[4] Kim, D. K.; Zhang, Y.; Voit, W.; Rao, K. V.; Muhammed, M.
Synthesis and characterization of surfactant-coated superparamagnetic monodispersed iron oxide nanoparticles. J. Magn. Magn. Mater. 2001, 225, 30-36.

[5] Lalatonne, Y.; Richardi, J.; Pileni, M. P. Van der Waals versus dipolar forces controlling mesoscopic organizations of magnetic nanocrystals. Nat. Mater. 2004, 3, 121-125.

[6] Gupta, A. K.; Gupta, M. Synthesis and surface engineering of iron oxide nanoparticles for biomedical applications. Biomaterials $\mathbf{2 0 0 5}$, 26, 3995-4021.

[7] Ali, A.; Zafar, H.; Zia, M.; ul Haq, I.; Phull, A. R.; Ali, J. S.; Hussain, A. Synthesis, characterization, applications, and challenges of iron oxide nanoparticles. Nanotechnol. Sci. Appl. 2016, 9, 49-67.

[8] Mansell, R.; Vemulkar, T.; Petit, D. C. M. C.; Cheng, Y.; Murphy, J.; Lesniak, M. S.; Cowburn, R. P. Magnetic particles with perpendicular anisotropy for mechanical cancer cell destruction. Sci. Rep. 2017, 7, 4257.

[9] Vemulkar, T.; Welbourne, E. N.; Mansell, R.; Petit, D. C. M. C.; Cowburn, R. P. The mechanical response in a fluid of synthetic antiferromagnetic and ferrimagnetic microdiscs with perpendicular magnetic anisotropy. Appl. Phys. Lett. 2017, 110, 042402.

[10] Gratton, S. E. A.; Ropp, P. A.; Pohlhaus, P. D.; Luft, J. C.; Madden, V. J.; Napier, M. E.; DeSimone, J. M. The effect of particle design on cellular internalization pathways. Proc. Natl. Acad. Sci. USA 2008, 105, 11613-11618.

[11] Parakhonskiy, B.; Zyuzin, M. V.; Yashchenok, A.; Carregal-Romero, S.; Rejman, J.; Möhwald, H.; Parak, W. J.; Skirtach, A. G. The influence of the size and aspect ratio of anisotropic, porous $\mathrm{CaCO}_{3}$ particles on their uptake by cells. J. Nanobiotechnol. 2015, 13, 53.

[12] Fredriksson, H.; Alaverdyan, Y.; Dmitriev, A.; Langhammer, C.; Sutherland, D. S.; Zäch, M.; Kasemo, B. Hole-mask colloidal lithography. Adv. Mater. 2007, 19, 4297-4302.

[13] Goiriena-Goikoetxea, M.; García-Arribas, A.; Rouco, M.; Svalov, A. V.; Barandiaran, J. M. High-yield fabrication of $60 \mathrm{~nm}$ permalloy nanodiscs in well-defined magnetic vortex state for biomedical applications. Nanotechnology 2016, 27, 175302.

[14] Chou, S. Y.; Krauss, P. R.; Zhang, W.; Guo, L. J.; Zhuang, L. Sub-10 nm imprint lithography and applications. J. Vac. Sci. Technol. B 1997, 15, 2897-2904.

[15] Heidari, B.; Maximov, I.; Sarwe, E. L.; Montelius, L. Large scale nanolithography using nanoimprint lithography. J. Vac. Sci. Technol. B 1999, 17, 2961-2964.

[16] Hu, W.; Wilson, R. J.; Koh, A.; Fu, A. H.; Faranesh, A. Z.; Earhart, C. M.; Osterfeld, S. J.; Han, S. J.; Xu, L.; Guccione, S. et al. High-moment antiferromagnetic nanoparticles with tunable magnetic properties. Adv. Mater. 2008, 20, 1479-1483.

[17] Hu, W.; Wilson, R. J.; Earhart, C. M.; Koh, A. L.; Sinclair, R.; Wang, S. X. Synthetic antiferromagnetic nanoparticles with tunable susceptibilities. J. Appl. Phys. 2009, 105, 07B508.

[18] Tiberto, P.; Barrera, G.; Celegato, F.; Conta, G.; Coïsson, M.; Vinai, F.; Albertini, $\mathrm{F}$. $\mathrm{Ni}_{80} \mathrm{Fe}_{20}$ nanodisks by nanosphere lithography for biomedical applications. J. Appl. Phys. 2015, 117, $17 \mathrm{~B} 304$.

[19] Chandramohan, A.; Sibirev, N. V.; Dubrovskii, V. G.; Petty, M. C.; Gallant, A. J.; Zeze, D. A. Model for large-area monolayer coverage of polystyrene nanospheres by spin coating. Sci. Rep. 2017, 7, 40888.

[20] Johnson, M. T.; Bloemen, P. J. H.; den Broeder, F. J. A.; de Vries, J. J. Magnetic anisotropy in metallic multilayers. Rep. Prog. Phys. 1996, 59, 1409.

[21] Bandiera, S.; Sousa, R. C.; Auffret, S.; Rodmacq, B.; Dieny, B. Enhancement of perpendicular magnetic anisotropy thanks to $\mathrm{Pt}$ insertions in synthetic antiferromagnets. Appl. Phys. Lett. 2012, 101, 072410 .

[22] Ruderman, M. A.; Kittel, C. Indirect exchange coupling of nuclear magnetic moments by conduction electrons. Phys. Rev. 1954, 96, 99-102.

[23] Kasuya, T. A theory of metallic ferro- and antiferromagnetism on zener's model. Prog. Theor. Phys. 1956, 16, 45-57.

[24] Yosida, K. Magnetic properties of Cu-Mn alloys. Phys. Rev. 1957, 106, 893-898.

[25] Bruno, P.; Chappert, C. Ruderman-kittel theory of oscillatory interlayer exchange coupling. Phys. Rev. B 1992, 46, 261-270. 
[26] Joisten, H.; Courcier, T.; Balint, P.; Sabon, P.; Faure-Vincent, J.; Auffret, S.; Dieny, B. Self-polarization phenomenon and control of dispersion of synthetic antiferromagnetic nanoparticles for biological applications. Appl. Phys. Lett. 2010, 97, 253112.

[27] Leulmi, S.; Joisten, H.; Dietsch, T.; Iss, C.; Morcrette, M.; Auffret, S.; Sabon, P.; Dieny, B. Comparison of dispersion and actuation properties of vortex and synthetic antiferromagnetic particles for biotechnological applications. Appl. Phys. Lett. 2013, 103, 132412.

[28] Lavrijsen, R.; Fernández-Pacheco, A.; Petit, D.; Mansell, R.; Lee, J. H.; Cowburn, R. P. Tuning the interlayer exchange coupling between single perpendicularly magnetized $\mathrm{CoFeB}$ layers. Appl. Phys. Lett. 2012, 100, 052411.

[29] Lee, J. H.; Mansell, R.; Petit, D.; Fernández-Pacheco, A.; Lavrijsen, R.; Cowburn, R. P. Domain imaging during soliton propagation in a 3D magnetic ratchet. SPIN 2013, 3, 1340013.

[30] Moore, T. A.; Miron, I. M.; Gaudin, G.; Serret, G.; Auffret, S.; Rodmacq, B.; Schuhl, A.; Pizzini, S.; Vogel, J.; Bonfim, M. High domain wall velocities induced by current in ultrathin $\mathrm{Pt} / \mathrm{Co} / \mathrm{AlO}_{x}$ wires with perpendicular magnetic anisotropy. Appl. Phys. Lett. 2008, 93,262504 . 